Supporting Information

\title{
Effect of Oxygen Vacancies on the Mechanoluminescence Response of Magnesium Oxide
}

Ekaterina Novitskaya, Aaron Manheim, Manuel Herrera*, and Olivia A. Graeve*

\section{Author Information}

\section{Corresponding Authors}

Olivia A. Graeve - Department of Mechanical and Aerospace Engineering, University of California San Diego, La Jolla, CA 92093-0411, United States; orcid.org/0000-00033599-0502; Email: ograeve@ucsd.edu; URL: http:/graeve.ucsd.edu/

Manuel Herrera - Centro de Nanociencias y Nanotecnología, Universidad Nacional Autónoma de México Ensenada, Baja California, México; orcid.org/0000-0002-7179654X; Email: zaldivar@cnyn.unam.mx

\section{Authors}

Ekaterina Novitskaya - Department of Mechanical and Aerospace Engineering, University of California San Diego, La Jolla, CA 92093-0411, United States

Aaron Manheim - Department of Mechanical and Aerospace Engineering, University of California San Diego, La Jolla, CA 92093-0411, United States

\section{UC San Diego}


Table S1. Impurities reported on the certificates of analysis for commercial MgO powders. Values reported in at.\% unless otherwise noted.

MgO(1): Product No.: 43195, Lot No.: L02Z039 (Alfa Aesar)

MgO(2): Product No.: 44078, Lot No.: D22D001 (Alfa Aesar)

MgO(3): Product No.: 44733, Lot No.: P22F020 (ThermoFisher Scientific)

MgO(4): Product No.: 41648, Lot No.: Y21E010 (ThermoFisher Scientific)

MgO(5): Product No.: 10800, Lot No.: Z06D039 (ThermoFisher Scientific)

\begin{tabular}{|c|c|c|c|c|c|c|}
\hline & $\mathbf{C a}$ & $\mathbf{C l}$ & $\mathbf{S}$ & $\mathbf{A l}$ & $\mathbf{F e}$ & $\mathbf{Z n}$ \\
\hline $\mathrm{MgO}(1)$ & 0.1 & & & 0.02 & 0.02 & 0.002 \\
\hline $\mathrm{MgO}(2)$ & $\begin{array}{c}0.83 \\
(\mathrm{CaO})\end{array}$ & 0.23 & & & & \\
\hline $\mathrm{MgO}(3)$ & $\begin{array}{c}<0.8 \\
(\mathrm{CaO})\end{array}$ & $<0.25$ & $\begin{array}{c}<0.45 \\
\left(\mathrm{SO}_{3}\right)\end{array}$ & $\begin{array}{c}<0.15 \\
\left(\mathrm{Al}_{2} \mathrm{O}_{3}\right)\end{array}$ & $\begin{array}{c}<0.15 \\
\left(\mathrm{~F}_{2} \mathrm{O}_{3}\right)\end{array}$ & $\begin{array}{c}<0.4 \\
(\mathrm{ZnO})\end{array}$ \\
\hline $\mathrm{MgO}(4)$ & & & & $50 \mathrm{ppm}$ & $7 \mathrm{ppm}$ \\
\hline $\mathrm{MgO}(5)$ & $3 \mathrm{ppm}$ & & & $<1 \mathrm{ppm}$ & $2 \mathrm{ppm}$ & $<1 \mathrm{ppm}$ \\
\hline
\end{tabular}

\begin{tabular}{|c|c|c|c|c|c|c|}
\hline & Mn & $\mathrm{Cr}$ & $\mathbf{T i}$ & $\mathbf{N a}$ & $\mathbf{K}$ & Other \\
\hline $\operatorname{MgO}(1)$ & 0.002 & $<0.0001$ & 0.0003 & & & $\begin{array}{c}\text { Pb: } 0.0006 \\
\text { Sn: } 0.0009 \\
\text { V: } 0.0007 \\
\text { Zr. } 0.002 \\
\text { B: } 0.004 \\
\text { Cd: }<0.0002 \\
\text { Mo: }<0.0003 \\
\text { Si: } 0.1\end{array}$ \\
\hline $\mathrm{MgO}(3)$ & $\begin{array}{l}<0.15 \\
(\mathrm{MnO})\end{array}$ & & $\begin{array}{l}<0.15 \\
\left(\mathrm{TiO}_{2}\right)\end{array}$ & $\begin{array}{l}<0.30 \\
\left(\mathrm{Na}_{2} \mathrm{O}\right)\end{array}$ & $\begin{array}{l}<0.05 \\
\left(\mathrm{~K}_{2} \mathrm{O}\right)\end{array}$ & $\begin{array}{l}\mathrm{Pb}:<6 \mathrm{ppm} \\
\mathrm{Hg}:<1 \mathrm{ppm}\end{array}$ \\
\hline $\mathrm{MgO}(4)$ & & & & & & $\begin{array}{l}\text { As: } 3 \mathrm{ppm} \\
\text { Be: } 8 \mathrm{ppm} \\
\mathrm{Ni}: 13 \mathrm{ppm} \\
\text { Pb: } 25 \mathrm{ppm} \\
\text { Sn: } 5 \mathrm{ppm}\end{array}$ \\
\hline $\operatorname{MgO}(5)$ & $<1 \mathrm{ppm}$ & $<1 \mathrm{ppm}$ & & $<1 \mathrm{ppm}$ & $<1$ ppm & $\begin{array}{l}\mathrm{Ag}:<1 \mathrm{ppm} \\
\mathrm{Au}:<1 \mathrm{ppm}\end{array}$ \\
\hline
\end{tabular}




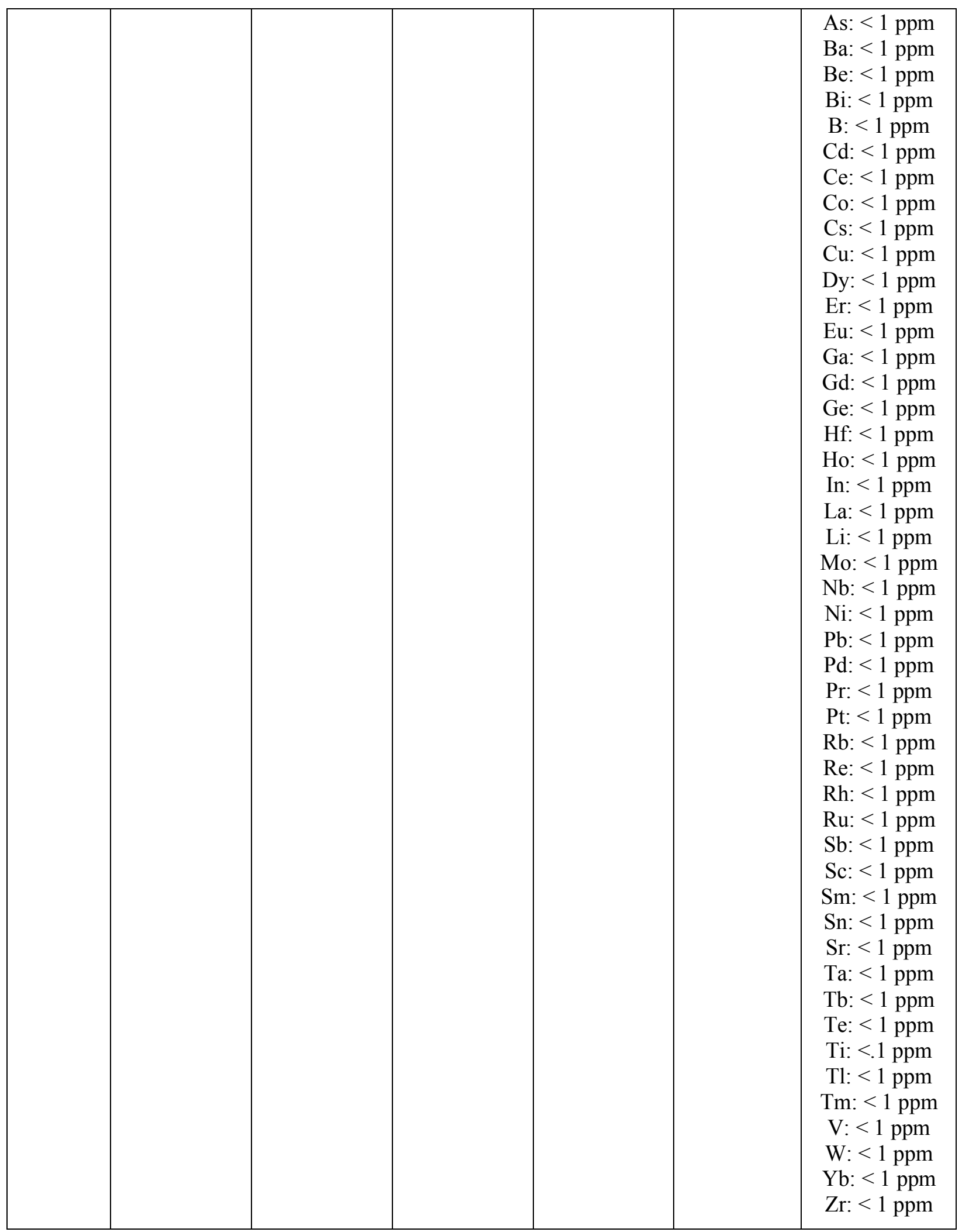


Table S2. Certificate of analysis for $\mathrm{Mg}\left(\mathrm{NO}_{3}\right)_{2}$ (Product No.: 11564; Lot No.: X17E071).

\begin{tabular}{|l|l|l|}
\hline Assay & $98.0-102.0 \%$ & $99.89 \%$ \\
\hline pH of a $\%$ solution & $5.0-8.2$ at $25{ }^{\circ} \mathrm{C}$ & 6.26 \\
\hline Insoluble matter & $0.005 \% \max$ & $<0.003 \%$ \\
\hline Chloride & $0.001 \% \max$ & $<0.001 \%$ \\
\hline Phosphate & $0.0005 \% \max$ & $<5 \mathrm{ppm}$ \\
\hline Sulfate & $0.005 \% \max$ & $<0.005 \%$ \\
\hline Barium & $0.005 \% \max$ & $<0.003 \%$ \\
\hline Ammonium & $0.003 \% \max$ & $<0.003 \%$ \\
\hline Calcium & $0.01 \% \max$ & None detected \\
\hline Heavy metals $(\mathrm{as} \mathrm{Pb})$ & $0.0005 \% \max$ & $<5 \mathrm{ppm}$ \\
\hline Iron & $0.0005 \% \max$ & $<5 \mathrm{ppm}$ \\
\hline Manganese & $0.0005 \% \max$ & $0.5 \mathrm{ppm}$ \\
\hline Potassium & $0.005 \% \max$ & $0.0007 \%$ \\
\hline Sodium & $0.005 \% \max$ & $<0.0018 \%$ \\
\hline Strontium & $0.005 \% \max$ & $0.0004 \%$ \\
\hline
\end{tabular}



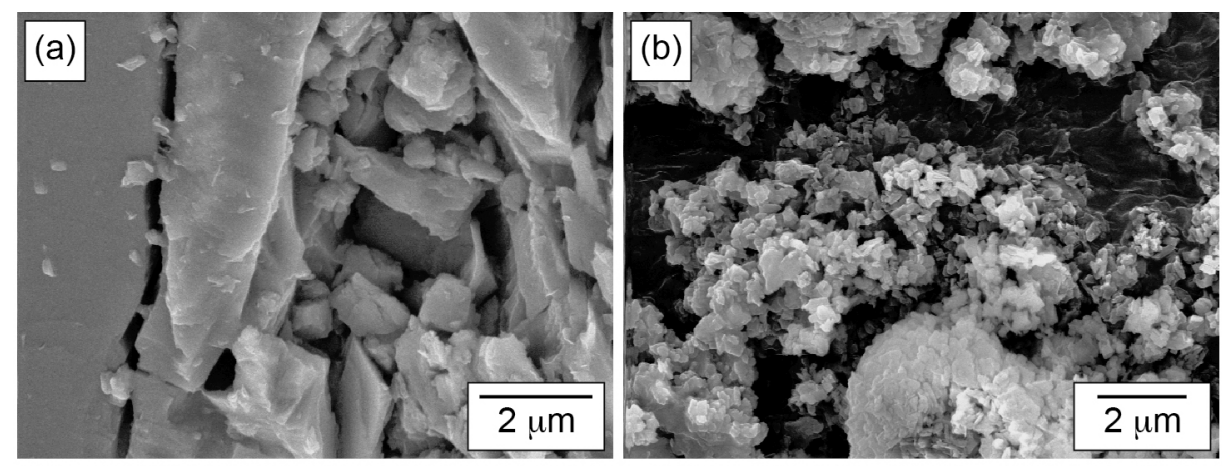

Figure S1.

Scanning electron micrographs of
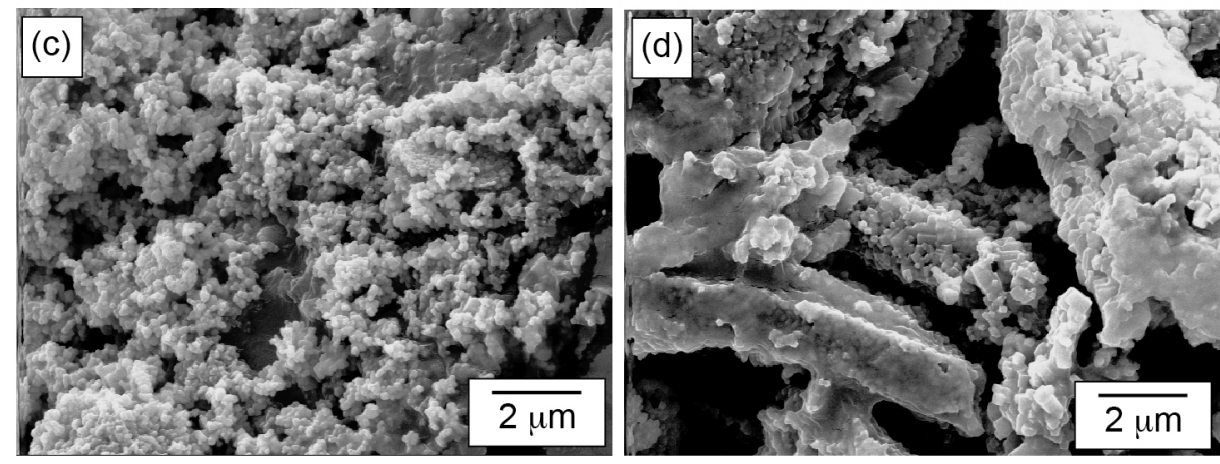
(a) $\mathrm{MgO}(1)$,
(b) $\mathrm{MgO}(2)$,
(c) $\mathrm{MgO}(3)$,
(d) $\mathrm{MgO}$ (4),
(e) $\operatorname{MgO}(5)$, and
(f) $\mathrm{MgO}$ (SCS).
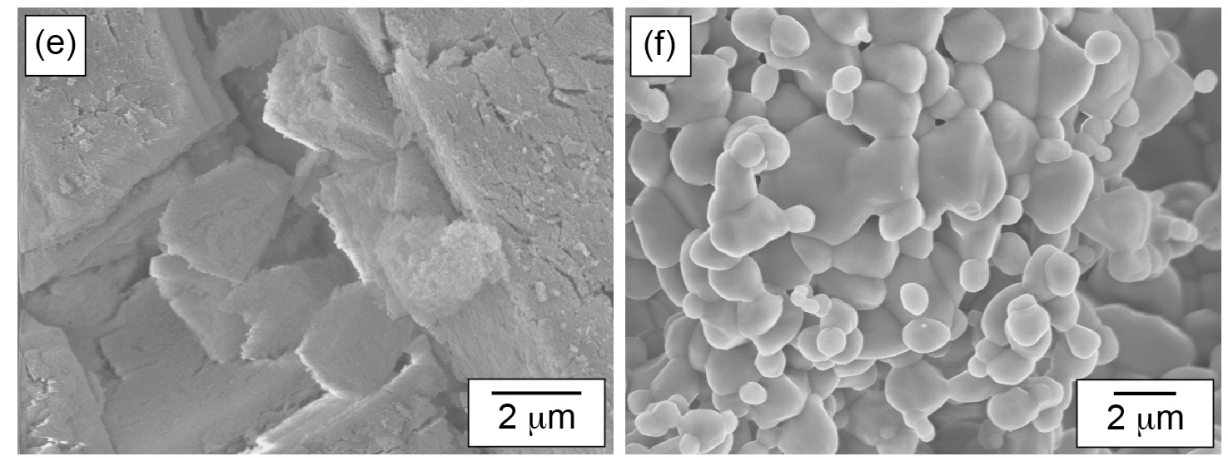


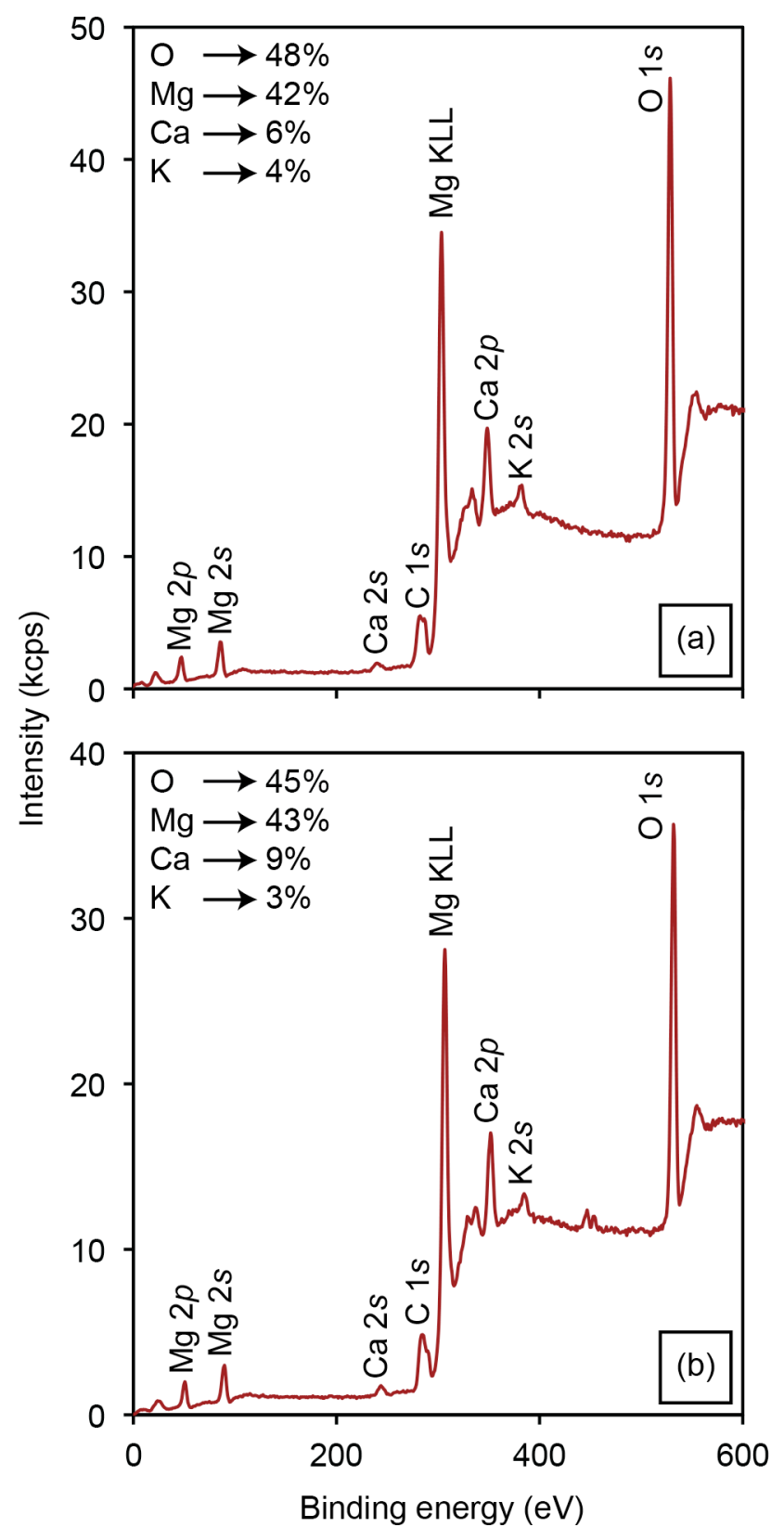

Figure S2. Survey X-ray photoelectron spectra for (a) $\mathrm{MgO}$ (1) and (b) $\mathrm{MgO}$ (SCS) powders, showing the presence of $\mathrm{Mg}, \mathrm{O}$, $\mathrm{Ca}$, and $\mathrm{K}$. 

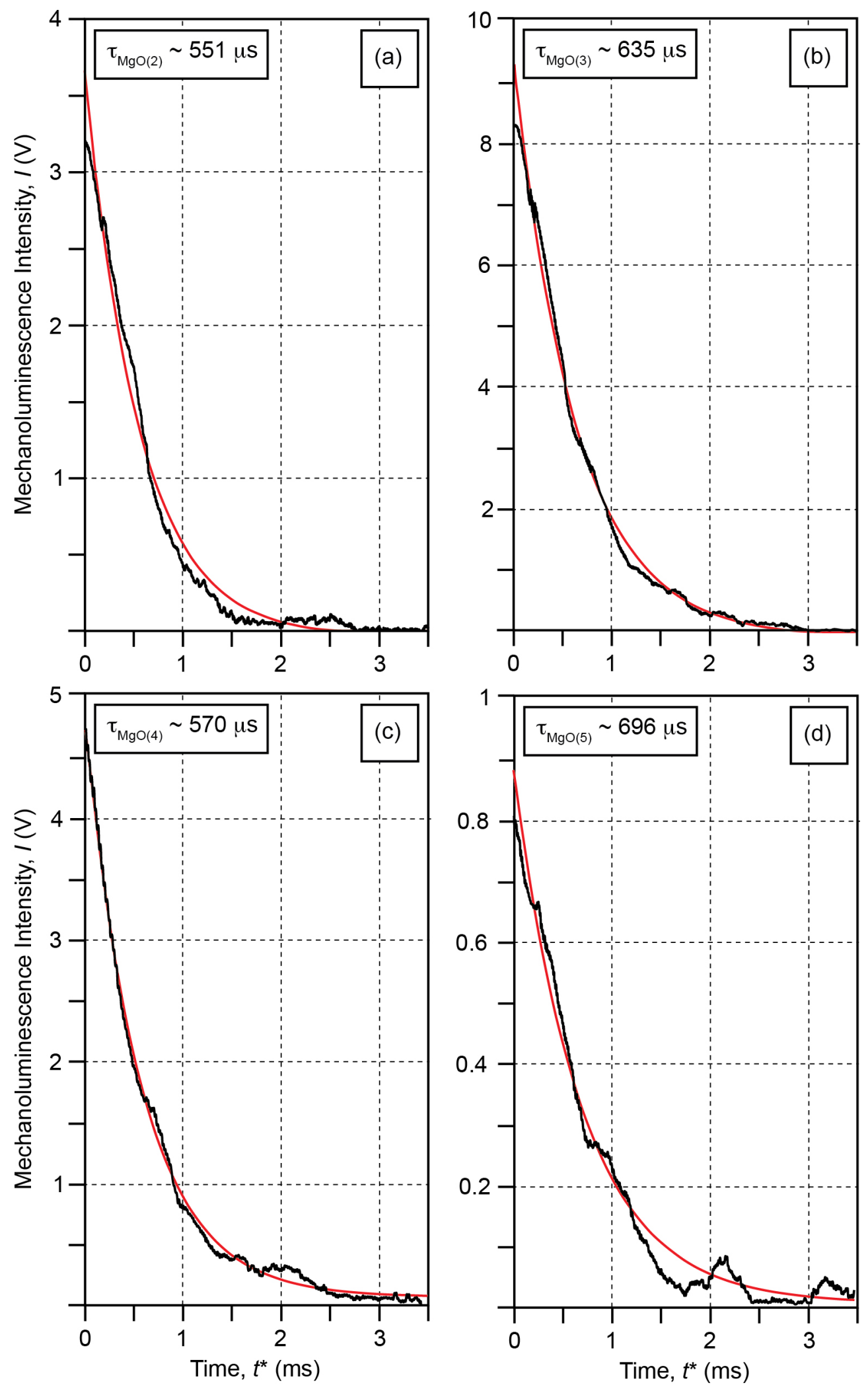

Figure S3.

Decay time curves for the

(a) $\mathrm{MgO}(2)$,

(b) $\mathrm{MgO}$ (3),

(c) $\mathrm{MgO}(4)$ and

(d) $\mathrm{MgO}(5)$ powders, calculated using the KohlrauschWilliam-Watts function; $t^{*}$ represents the time at which decay is initiated. The coefficients of determination, $\mathrm{R}^{2}$, of the fittings were 0.9865 for $\mathrm{MgO}(2)$, 0.9950 for $\mathrm{MgO}(3)$, 0.9971 for $\mathrm{MgO}(4)$, and 0.9833 for $\mathrm{MgO}(5)$. 